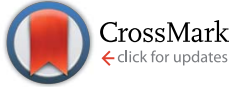

Cite this: RSC Adv., 2016, 6, 39335

Received 19th February 2016 Accepted 4th April 2016

DOI: $10.1039 / c 6 r a 04524 c$

www.rsc.org/advances

\section{Phosphine-free chiral iridium catalysts for asymmetric catalytic hydrogenation of simple ketones $\uparrow$}

\author{
Prashant Kumar, ${ }^{\star a b}$ Torsten Irrgang, ${ }^{b}$ George E. Kostakis ${ }^{a}$ and Rhett Kempe ${ }^{\star b}$
}

Novel pyridylalkylamine and aminopyridinato ligand stabilized iridium complexes with no P ligand are introduced. These complexes have been investigated as catalysts for asymmetric hydrogenation of simple ketones, resulting in an active catalyst for bulky alkyl aryl ketones that is $\alpha$-methylpropiophenone. The ligands were synthesized from inexpensive starting materials and their modular design allows for the introduction of a broad variety of substitution patterns. Additionally, better activity and selectivity was observed at $20{ }^{\circ} \mathrm{C}$ and 20 bar $\mathrm{H}_{2}$ pressure with a catalyst loading as low as $0.05 \mathrm{~mol} \%$ iridium. These phosphorus free catalysts have always been a central issue in both academic and industrial research.

\section{Introduction}

The catalytic reduction of polar multiple bonds-mainly carbonyl $(-\mathrm{C}=\mathrm{O})$ functionalities into corresponding hydroxyl $(-\mathrm{CH}-\mathrm{OH})$ functionalities by molecular hydrogen has great significance in modern synthetic chemistry. The stereoselective hydrogenation of ketones to yield enantiomerically pure alcohols is a key step, in the synthesis of fine chemicals, perfumes, and pharmaceuticals. This reaction is normally performed by complexes of precious metals (e.g. Ru, Rh, Ir) using either $\mathrm{H}_{2}$ or iPrOH as the hydrogen source. ${ }^{1}$ In particular, the reduction of ketones with gaseous hydrogen provides an atom-economical synthetic method. High enantioselectivity, low catalyst loadings, quantitative yields, atom economy and mild conditions are attractive features of this transformation as evident in the ever growing list of research articles that use these methods. The importance of this topic was recognized when Knowles ${ }^{2,3}$ and Noyori ${ }^{3,4}$ were awarded the 2001 Nobel prize for their instrumental contributions to this field. The development of highly enantioselective chemocatalyst based on their low toxicity and lower price ligands could drastically increase the importance of asymmetric homogeneous catalysis. ${ }^{5}$ Therefore, it has been well established that organometallic complexes of ruthenium have been wellemployed in this scenario, recently in particular by the groups of Noyori ${ }^{6}$ and Braunstein. ${ }^{7}$ Iridium and rhodium complexes have long been known to be highly effective homogeneous

${ }^{a}$ Department of Chemistry, School of Life Sciences, University of Sussex, Brighton BN1 9QJ, UK. E-mail: pbhukumar@gmail.com

${ }^{b}$ Inorganic Chemistry II, University of Bayreuth, 95440-Bayreuth, Germany. E-mail: kempe@uni-bayreuth.de

$\dagger$ Electronic supplementary information (ESI) available. CCDC 1437480-1437484 and 1473406. For ESI and crystallographic data in CIF or other electronic format see DOI: $10.1039 / \mathrm{c} 6 \mathrm{ra} 04524 \mathrm{c}$ hydrogenation catalysts, with $\mathrm{RhCl}\left(\mathrm{PPh}_{3}\right)_{3}$ (Wilkinson's catalyst $)^{8}$ and the most widely used $\left[\operatorname{Ir}(\operatorname{cod})(\mathrm{py})\left(\mathrm{PCy}_{3}\right)\right] \mathrm{PF}_{6}[\mathrm{py}=$ pyridine, $\operatorname{cod}=1,5$-cyclooctadiene, Crabtree's catalyst $\left.{ }^{9}\right]$. In contrast, iridium phosphorus-free catalysts are extensively less efficient and/or enantioselective in this asymmetric hydrogenation. ${ }^{10}$ Relatively, few organometallic complexes derived from amine based ligands have been reported to be effective at the control of asymmetric hydrogenation reactions, ${ }^{\mathbf{1 0 , 1 1}}$ particularly in comparison to the large numbers of diphosphine ${ }^{12,13}$ and mixed amine/phosphine ${ }^{\mathbf{1 4 , 1 5}}$ ligands, which have been reported. In principle, amine-based ligands possess a potential advantage over phosphorus because they are relatively simple to prepare and less prone to decomposition and oxidation reactions. Furthermore, iridium(I) complexes of cycloocta-1,5-diene (cod) are of interest because the coordinated cod can be replaced by other ligands. Cod can also be hydrogenated to provide vacant coordination sites around iridium and thus increase the catalytic activities of the complexes. ${ }^{16}$ In this paper, we report the synthesis and structure of phosphorus-free iridium catalysts containing aminopyridinato and pyridylalkylamine (Scheme 1) and cod ligands for the hydrogenation of simple ketones.<smiles>Cc1cccc(NC(CO)CC(C)C)n1</smiles><smiles>CC(C)CC(CO)Nc1ccccn1</smiles><smiles>OCC(Cc1ccccc1)NCc1ccccn1</smiles><smiles>CC(CO)NCc1ccccn1</smiles><smiles>Cc1cccc(CNC(CO)Cc2ccccc2)n1</smiles><smiles>Cc1cccc(CNC(CO)CC(C)C)n1</smiles><smiles>Cc1cccc(CNC(C)CO)n1</smiles><smiles>Cc1cccc(CNC(CO)c2ccccc2)n1</smiles>

Scheme 1 Aminopyridinato (a, b) and pyridylalkylamine (c-h) ligands. 


\section{Experiment section}

\subsection{General method}

All reactions and manipulations which are sensitive to moisture or air were performed in argon-filled glove box or using standard Schlenk techniques. Non-halogenated solvents were distilled from sodium benzophenone ketyl and halogenated solvents from $\mathrm{P}_{2} \mathrm{O}_{5}$. Deuterated solvents were obtained from Cambridge Isotope Laboratories and were degassed, dried and distilled prior to use. $[\operatorname{Ir}(\mathrm{OMe})(\operatorname{cod})]_{2}(\operatorname{cod}=1,5$-cyclooctydiene, $\mathrm{Me}=$ methyl) was prepared according to the literature. ${ }^{17}$ All chemicals were purchased from commercial vendors. ${ }^{1} \mathrm{H}$ and ${ }^{13} \mathrm{C}$ NMR spectra were obtained at room temperature on ARX 250 or Varian INNOVA 300 or 400 spectrometer using $\mathrm{C}_{6} \mathrm{D}_{6}$, and $\mathrm{CDCl}_{3}$ as a solvent and chemical shifts are reported in ppm relative to the deuterated solvent. Elemental analyses for $\mathrm{C}, \mathrm{H}$ and $\mathrm{N}$ were performed on a Vario elementary EL III apparatus.

\subsection{Synthetic procedures}

2.2.1. Preparation of aminopyridinato ligands.

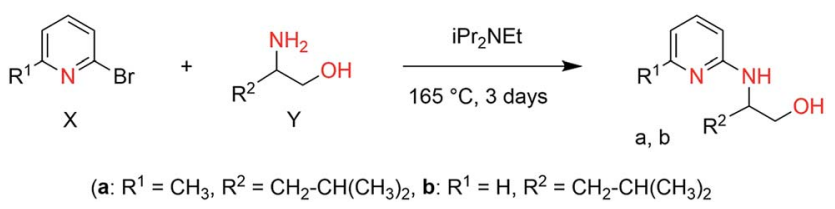

General procedure. A $25 \mathrm{~mL}$ medium-pressure tube charged with 2-bromopyridine $(1.00 \mathrm{mmol})$, or 6-methyl-2-bromopyridine $(1.00 \mathrm{mmol}) \mathrm{X}$, substituted amino alcohol (1.00 mmol) Y, $N, N$-diisopropyl ethylamine $(1.16 \mathrm{mmol})$ and a stir bar was flushed with nitrogen several times, stoppered and heated at $160 \pm 5{ }^{\circ} \mathrm{C}$ for 2 days. The tube was allowed to cool to room temperature, the contents was diluted with a small amount of $\mathrm{CH}_{2} \mathrm{Cl}_{2}$ and chromatographed (hexanes-EtOAc $2: 1 \rightarrow 1: 1 \rightarrow$ neat EtOAc) to afford the product $(\mathbf{a}, \mathbf{b})$ in good yield.

4-Methyl-2-(6-methyl-pyridin-2-yl-amino)-pentan-1-ol (a). Yellow viscous liquid, $64 \%$ yield. $\mathrm{C}_{12} \mathrm{H}_{20} \mathrm{~N}_{2} \mathrm{O}$ (208.16): calcd $\mathrm{C} 69.19, \mathrm{H}$ 9.68, N 13.45; found C 68.73, H 10.01, N 12.97. ${ }^{1} \mathrm{H}$ NMR $(250$ $\left.\mathrm{MHz} \mathrm{CDCl}_{3}, 298 \mathrm{~K}\right): \delta=7.26(\mathrm{t}, J=3.6 \mathrm{~Hz}, 1 \mathrm{H}, \mathrm{Ar}) ; 6.37$ (d, $J=$ $5.2 \mathrm{~Hz}, 1 \mathrm{H}, \mathrm{Ar}) ; 6.25$ (d, $J=4.5 \mathrm{~Hz}, 1 \mathrm{H}, \mathrm{Ar}) ; 3.78-3.70(\mathrm{~m}, 2 \mathrm{H}$, $\left.\mathrm{CH}_{2}-\mathrm{OH}\right)$; 3.49-3.45 (m, 1H, CH-NH); 2.29 (s, 3H, $\left.\mathrm{CH}_{3}\right), 1.69-$ $1.64\left(\mathrm{~m}, 1 \mathrm{H}, \mathrm{CH}-\left(\mathrm{CH}_{3}\right)_{2}\right) ; 1.45-1.30\left(\mathrm{~m}, 2 \mathrm{H}, \mathrm{CH}_{2}-\mathrm{CH}-\left(\mathrm{CH}_{3}\right)_{2}\right)$; $0.88-0.83\left(\mathrm{dd}, J=5.2 \mathrm{~Hz}, 6 \mathrm{H}, \mathrm{CH}-\left(\mathrm{CH}_{3}\right)_{2}\right) \mathrm{ppm} .{ }^{13} \mathrm{C} \mathrm{NMR}(62.90$ $\mathrm{MHz}, \mathrm{CDCl}_{3}, 298 \mathrm{~K}$ ): $\delta=158.14$ (C5, pyridine), 155.81 (C1, pyridine), 137.96 (C3, pyridine), 111.93 (C4, pyridine), 104.79 (C2, pyridine), $67.65\left(\mathrm{CH}_{2}, \mathrm{CH}_{2} \mathrm{OH}\right), 52.97$ ( $\left.\mathrm{CH}, \mathrm{CHNH}\right) ; 41.06$ $\left(\mathrm{CH}_{2}, \mathrm{CH}_{2} \mathrm{CH}\left(\mathrm{CH}_{3}\right)_{2}\right) ; 24.87\left(\mathrm{CH}-\left(\mathrm{CH}_{3}\right)_{2}\right) ; 23.69,22.93(\mathrm{CH}-$ $\left.\left(\mathrm{CH}_{3}\right)_{2}\right) ; 22.22\left(\mathrm{CH}_{3}\right)$ ppm.

4-Methyl-2-(pyridin-2-yl-amino)-pentan-1-ol (b). White solid, $40 \%$ yield. $\mathrm{C}_{11} \mathrm{H}_{18} \mathrm{~N}_{2} \mathrm{O}$ (194.14): calcd C 68.01, $\mathrm{H}$ 9.34, N 14.42; found C 67.86, $\mathrm{H}$ 9.21, N 14.41. ${ }^{1} \mathrm{H} \mathrm{NMR}\left(250 \mathrm{MHz}, \mathrm{CDCl}_{3}, 298\right.$ $\mathrm{K}): \delta=7.99(\mathrm{~d}, J=5.4 \mathrm{~Hz}, 1 \mathrm{H}, \mathrm{Ar}) ; 6.95(\mathrm{t}, J=7.4 \mathrm{~Hz}, 1 \mathrm{H}, \mathrm{Ar})$; $6.23(\mathrm{t}, J=5.5 \mathrm{~Hz}, 1 \mathrm{H}, \mathrm{Ar}) ; 5.94(\mathrm{~d}, J=7.4 \mathrm{~Hz}, 1 \mathrm{H}, \mathrm{Ar}) ; 3.66-3.61$ $\left(\mathrm{m}, 2 \mathrm{H}, \mathrm{CH}_{2}-\mathrm{OH}\right) ; 3.48-3.47(\mathrm{~m}, 1 \mathrm{H}, \mathrm{CH}-\mathrm{NH}) ; 1.48-1.46(\mathrm{~m}, 1 \mathrm{H}$, $\left.\mathrm{CH}-\left(\mathrm{CH}_{3}\right)_{2}\right) ; 1.14-1.08$ (m, 2H, $\left.\mathrm{CH}_{2}-\mathrm{CH}-\left(\mathrm{CH}_{3}\right)_{2}\right) ; 0.78-0.74$ (dd, $J$ $\left.=5.2 \mathrm{~Hz}, 6 \mathrm{H}, \mathrm{CH}-\left(\mathrm{CH}_{3}\right)_{2}\right) \mathrm{ppm} .{ }^{13} \mathrm{C} \mathrm{NMR}\left(62.90 \mathrm{MHz}, \mathrm{CDCl}_{3}\right.$,
$298 \mathrm{~K}$ ): $\delta=158.94$ (C5, pyridine); 147.88 (C1, pyridine); 137.03 (C3, pyridine); 112.71 (C2, pyridine); 108.38 (C4, pyridine); 52.23 $\left(\mathrm{CH}_{2}, \mathrm{CH}_{2} \mathrm{OH}\right) ; 44.99$ (CH, $\left.\mathrm{CHNH}\right) ; 41.07\left(\mathrm{CH}_{2}, \mathrm{CH}_{2} \mathrm{CH}\left(\mathrm{CH}_{3}\right)_{2}\right)$; $24.99\left(\mathrm{CH}-\left(\mathrm{CH}_{3}\right)_{2}\right) ; 22.18,23.05\left(\mathrm{CH}-\left(\mathrm{CH}_{3}\right)_{2}\right) \mathrm{ppm}$.

\subsubsection{Preparation of pyridylalkylamine ligands}

General procedure. To ice-cooled solution of amino alcohol (1.00 mmol) (Y), pyridine-2-carbaldehyde or 6-methylpyridine-2carbaldehyde $(1.00 \mathrm{mmol})\left(\mathbf{X}^{\prime}\right)$ was added slowly in $20 \mathrm{~mL}$ of methanol. The resulting solution was stirred at room temperature for $3 \mathrm{~h}$ and subsequently treated with sodiumtetrahydridoborate $(2.5 \mathrm{mmol})$ in small portions. After additional $2 \mathrm{~h}$, water $(50 \mathrm{~mL})$ was added and the reaction mixture was concentrated to about $10 \mathrm{~mL}$ using rotovap. The remaining solution was extracted with dichloromethane $(2 \times 20 \mathrm{~mL})$, and the organic layer was separated and subsequently dried with sodium sulphate and concentrated in vacuum to afford the product (c-h) in good to high yield.

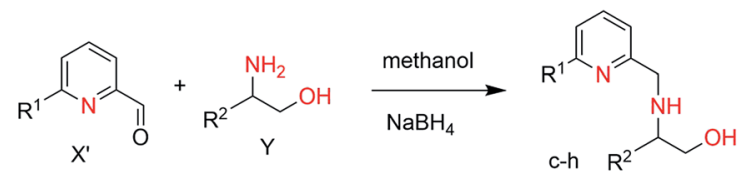

(c: $\mathrm{R}^{1}=\mathrm{H}, \mathrm{R}^{2}=\mathrm{CH}_{2}-\mathrm{C}_{6} \mathrm{H}_{5}, \mathbf{d}: \mathrm{R}^{1}=\mathrm{H}, \mathrm{R}^{2}=\mathrm{CH}_{3}, \mathbf{e}: \mathrm{R}^{1}=\mathrm{CH}_{3}, \mathrm{R}^{2}=\mathrm{CH}_{2}-\mathrm{C}_{6} \mathrm{H}_{5}$, f: $\left.R^{1}=\mathrm{CH}_{3}, \mathrm{R}^{2}=\mathrm{CH}_{2}-\mathrm{CH}\left(\mathrm{CH}_{3}\right)_{2}, \mathbf{g :} \mathrm{R}^{1}=\mathrm{CH}_{3}, \mathrm{R}^{2}=\mathrm{CH}_{3}, \mathbf{h}: \mathrm{R}^{1}=\mathrm{CH}_{3}, \mathrm{R}^{2}=\mathrm{C}_{6} \mathrm{H}_{5}\right)$

3-Phenyl-2-[(pyridin-2-yl-methyl)-amino]-propan-1-ol (c). Yellow solid, $90 \%$ yield. mp $65^{\circ} \mathrm{C}$. $\mathrm{C}_{15} \mathrm{H}_{18} \mathrm{~N}_{2} \mathrm{O}$ (242.14): calcd C 73.35, $\mathrm{H}$ 7.49, N 11.56; found $\mathrm{C} 74.43, \mathrm{H} 7.31, \mathrm{~N}$ 11.32. ${ }^{1} \mathrm{H}$ NMR (250 $\left.\mathrm{MHz} \mathrm{CDCl}_{3}, 298 \mathrm{~K}\right): \delta=8.46(\mathrm{~d}, J=1.4 \mathrm{~Hz}, 1 \mathrm{H}, \mathrm{Ar}), 7.56(\mathrm{t}, J=$ $3.8 \mathrm{~Hz}, 1 \mathrm{H}, \mathrm{Ar}), 7.24-7.08$ (m, 5H, Ar), 3.97-3.82 (m, 3H, $\mathrm{CH}_{2}-\mathrm{N}$, $\mathrm{CH}-\mathrm{N}), 3.63-3.58\left(\mathrm{~m}, 2 \mathrm{H}, \mathrm{CH}_{2}-\mathrm{O}\right), 2.73-2.95$ (m, 2H, $\left.\mathrm{CH}_{2}-\mathrm{Ar}\right)$ ppm. ${ }^{13} \mathrm{C}$ NMR $\left(62.90 \mathrm{MHz}, \mathrm{CDCl}_{3}, 298 \mathrm{~K}\right): \delta=160.11$ (C5, pyridine), 157.85 (C1, pyridine), 139.68 (C9, phenyl), 136.42 (C3, pyridine), 129.65 (C10, phenyl), 128.49 (C11, phenyl), 126.21 (C12, phenyl), 121.11 (C4, pyridine), 118.96 (C2, pyridine), 62.61 $\left(\mathrm{CH}_{2}, \mathrm{CH}_{2} \mathrm{OH}\right), 60.97(\mathrm{CH}, \mathrm{CHNH}), 52.31\left(\mathrm{CH}_{2}, \mathrm{CH}_{2} \mathrm{NH}\right), 38.66$ $\left(\mathrm{CH}_{2}, \mathrm{CH}_{2} \mathrm{C}_{6} \mathrm{H}_{5}\right)$ ppm.

2-[(Pyridin-2-yl-methyl)-amino]-propan-1-ol (d). Yellow solid, $90 \%$ yield. mp $70{ }^{\circ} \mathrm{C} . \mathrm{C}_{9} \mathrm{H}_{14} \mathrm{~N}_{2} \mathrm{O}$ (166.11): calcd C 65.03, H 8.49, $\mathrm{N}$ 16.85; found C 64.89, H 8.38, N 16.27. ${ }^{1} \mathrm{H}$ NMR $(250 \mathrm{MHz}$, $\left.\mathrm{CDCl}_{3}, 298 \mathrm{~K}\right): \delta=8.38(\mathrm{~d}, J=1.8 \mathrm{~Hz}, 1 \mathrm{H}) ; 7.06(\mathrm{t}, J=5.2,1 \mathrm{H})$; $6.91(\mathrm{~d}, J=3.6,1 \mathrm{H}) ; 6.60(\mathrm{t}, J=6.5 \mathrm{~Hz}, 1 \mathrm{H}) ; 3.85-3.70(\mathrm{~m}, 2 \mathrm{H}$, $\left.\mathrm{CH}_{2}-\mathrm{N}\right)$; 3.55-3.52 (m, 2H, $\left.\mathrm{CH}_{2}-\mathrm{O}\right) ; 2.70-2.66$ (m, $\left.1 \mathrm{H}, \mathrm{CH}-\mathrm{NH}\right)$; $0.92\left(\mathrm{~d}, J=3.2,3 \mathrm{H}, \mathrm{CH}_{3}\right) .{ }^{13} \mathrm{C} \mathrm{NMR}\left(62.90 \mathrm{MHz}, \mathrm{CDCl}_{3}, 298 \mathrm{~K}\right)$ : $\delta=160.53$ (C5, pyridine); 149.176 (C1, pyridine); 136.00 (C3, pyridine); 122.11 (C4, pyridine); 121.67 (C2, pyridine); 65.63 $\left(\mathrm{CH}_{2}, \mathrm{CH}_{2} \mathrm{OH}\right) ; 54.70(\mathrm{CH}, \mathrm{CHNH}) ; 52.33\left(\mathrm{CH}_{2}, \mathrm{CH}_{2} \mathrm{NH}\right) ; 17.16$ $\left(\mathrm{CH}_{3}, \mathrm{CH}_{3} \mathrm{CH}\right)$ ppm.

2-[(6-Methyl-pyridin-2-yl-methyl)-amino]-3-phenyl-propan-1-ol (e). Yellow solid, $91 \%$ yield. mp $76{ }^{\circ} \mathrm{C} . \mathrm{C}_{16} \mathrm{H}_{20} \mathrm{~N}_{2} \mathrm{O}$ (256.16): calcd $\mathrm{C}$ 73.86, H 7.86, N 10.93; found C 73.96, H 7.69, N 10.68. ${ }^{1} \mathrm{H}$ NMR $\left(250 \mathrm{MHz}, \mathrm{CDCl}_{3}, 298 \mathrm{~K}\right): \delta=7.44(\mathrm{t}, J=1.4 \mathrm{~Hz}, 1 \mathrm{H}, \mathrm{Ar}), 7.29-7.16$ (m, 5H, Ar), 6.92 (t, J=3.8 Hz, 1H, Ar), 3.91-3.82 (m, 3H, $\mathrm{CH}_{2}-\mathrm{N}$, CH-N), 3.60-3.41 (m, 2H, $\mathrm{CH}_{2}-\mathrm{O}$ ), 2.91-2.79 (m, 2H, $\left.\mathrm{CH}_{2}-\mathrm{Ar}\right), 2.48$ (s, 3H, $\mathrm{CH}_{3}$ ) ppm. ${ }^{13} \mathrm{C} \mathrm{NMR}\left(62.90 \mathrm{MHz}, \mathrm{CDCl}_{3}, 298 \mathrm{~K}\right): \delta=157.84$ (C5, pyridine), 158.83 (C1, pyridine), 138.88 (C9, phenyl), 136.93 (C3, pyridine), 129.29 (C10, phenyl), 128.43 (C11, phenyl), 126.24 
(C12, phenyl), 121.63 (C4, pyridine), 119.13 (C2, pyridine), 62.57 $\left(\mathrm{CH}_{2}, \mathrm{CH}_{2} \mathrm{OH}\right), 60.75(\mathrm{CH}, \mathrm{CHNH}), 51.93\left(\mathrm{CH}_{2}, \mathrm{CH}_{2} \mathrm{NH}\right), 38.24$ $\left(\mathrm{CH}_{2}, \mathrm{CH}_{2} \mathrm{C}_{6} \mathrm{H}_{5}\right), 24.19\left(\mathrm{CH}_{3}\right)$ ppm.

4-Methyl-2-[(6-methyl-pyridin-2-yl-methyl)-amino]-propan-1-ol (f). Pale yellow solid, $94 \%$ yield. $\mathrm{mp} 60^{\circ} \mathrm{C}$. $\mathrm{C}_{13} \mathrm{H}_{22} \mathrm{~N}_{2} \mathrm{O}$ (222.17): calcd C 70.23, H 9.97, N 12.60; found C 70.44, H 10.20, N 12.51. ${ }^{1} \mathrm{H}$ NMR (250 MHz, $\left.\mathrm{CDCl}_{3}, 298 \mathrm{~K}\right): \delta=7.23(\mathrm{~d}, J=2.4 \mathrm{~Hz}, 1 \mathrm{H}, \mathrm{Ar})$, $6.63(\mathrm{~d}, J=4.3 \mathrm{~Hz}, 1 \mathrm{H}, \mathrm{Ar}), 3.88-3.83$ (d, $\left.J=5.4,2 \mathrm{H}, \mathrm{CH}_{2}-\mathrm{NH}\right)$; 3.83-3.74 (dd, $1 \mathrm{H}, \mathrm{CH}-\mathrm{NH}$ ); 3.49-3.43 (dd, $\left.1 \mathrm{H}, \mathrm{CH}_{2}-\mathrm{OH}\right) ; 2.80-$ 2.49 (m, $\left.1 \mathrm{H}, \mathrm{CH}_{2}-\mathrm{OH}\right) ; 2.72\left(\mathrm{~s}, 3 \mathrm{H}, \mathrm{CH}_{3}\right), 1.78-1.67$ (sept, $1 \mathrm{H}, \mathrm{CH}-$ $\left.\left(\mathrm{CH}_{3}\right)_{2}\right)$; 1.45-1.30 (m, 2H, $\left.\mathrm{CH}_{2}-\mathrm{CH}-\left(\mathrm{CH}_{3}\right)_{2}\right) ; 0.86-0.82(\mathrm{t}, J=6.4$ $\left.\mathrm{Hz}, 6 \mathrm{H}, \mathrm{CH}-\left(\mathrm{CH}_{3}\right)_{2}\right)$ ppm. ${ }^{13} \mathrm{C} \mathrm{NMR}\left(62.90 \mathrm{MHz}, \mathrm{CDCl}_{3}, 298 \mathrm{~K}\right)$ : $\delta=160.20$ (C5, pyridine), 157.91 (C1, pyridine), 136.59 (C3, pyridine), 121.26 (C4, pyridine), $119.32\left(\mathrm{C} 2\right.$, pyridine), $63.62\left(\mathrm{CH}_{2}\right.$, $\left.\mathrm{CH}_{2} \mathrm{OH}\right), 57.35(\mathrm{CH}, \mathrm{CHNH}), 52.21\left(\mathrm{CH}_{2}, \mathrm{CH}_{2} \mathrm{NH}\right) ; 41.61\left(\mathrm{CH}_{2}\right.$, $\left.\mathrm{CH}_{2} \mathrm{CH}\left(\mathrm{CH}_{3}\right)_{2}\right) ; 25.06\left(\mathrm{CH}-\left(\mathrm{CH}_{3}\right)_{2}\right) ; 24.20,23.15\left(\mathrm{CH}-\left(\mathrm{CH}_{3}\right)_{2}\right)$; $22.91\left(\mathrm{CH}_{3}\right) \mathrm{ppm}$.

2-[(6-Methyl-pyridin-2-yl-methyl)-amino]-propan-1-ol (g). Brown solid, $84 \%$ yield. $m p 75{ }^{\circ} \mathrm{C} . \mathrm{C}_{10} \mathrm{H}_{16} \mathrm{~N}_{2} \mathrm{O}$ (180.13): calcd C 66.63, $\mathrm{H}$ 8.95, N 15.54; found C 66.38, H 8.64, N 15.09. ${ }^{1} \mathrm{H}$ NMR (250 $\left.\mathrm{MHz}, \mathrm{CDCl}_{3}, 298 \mathrm{~K}\right): \delta=7.04(\mathrm{t}, J=2.6 \mathrm{~Hz}, 1 \mathrm{H}) ; 6.79(\mathrm{~d}, J=3.6$, $1 \mathrm{H}) ; 6.55(\mathrm{~d}, J=4.5 \mathrm{~Hz}, 1 \mathrm{H}) ; 3.85-3.70\left(\mathrm{~m}, 2 \mathrm{H}, \mathrm{CH}_{2}-\mathrm{N}\right) ; 3.37-$ 3.35 (m, 2H, $\left.\mathrm{CH}_{2}-\mathrm{O}\right)$; 2.71-2.69 (m, 1H, CH-NH); 2.35 (s, 3H, $\mathrm{CH}_{3}$ ); 0.94 (dd, 3H, $\mathrm{CH}_{3}$ ) ppm. ${ }^{13} \mathrm{C} \mathrm{NMR}\left(62.90 \mathrm{MHz}, \mathrm{CDCl}_{3}, 298\right.$ $\mathrm{K}): \delta=159.91$ (C5, pyridine); 157.98 (C1, pyridine); 136.45 (C3, pyridine); 121.14 (C4, pyridine); 119.39 (C2, pyridine); 65.49 $\left(\mathrm{CH}_{2}, \mathrm{CH}_{2} \mathrm{OH}\right) ; 55.02(\mathrm{CH}, \mathrm{CHNH}) ; 52.50\left(\mathrm{CH}_{2}, \mathrm{CH}_{2} \mathrm{NH}\right) ; 23.99$ $\left(\mathrm{CH}_{3}\right) ; 17.21\left(\mathrm{CH}_{3}, \mathrm{CH}_{3} \mathrm{CH}\right)$ ppm.

2-[(6-Methyl-pyridin-2-yl-methyl)-amino]-2-phenyl-ethanol (h). Yellow solid, 84\% yield. mp $60{ }^{\circ} \mathrm{C} . \mathrm{C}_{15} \mathrm{H}_{18} \mathrm{~N}_{2} \mathrm{O}$ (242.14): calcd C 74.35, H 7.49, N 11.56; found C 74.23, H 7.07, N 11.56. ${ }^{1} \mathrm{H}$ NMR $\left(250 \mathrm{MHz}, \mathrm{CDCl}_{3}, 298 \mathrm{~K}\right): \delta=7.44(\mathrm{t}, J=1.4 \mathrm{~Hz}, 1 \mathrm{H}, \mathrm{Ar}), 7.29-$ $7.16(\mathrm{~m}, 5 \mathrm{H}, \mathrm{Ar}), 6.92(\mathrm{t}, J=3.8 \mathrm{~Hz}, 1 \mathrm{H}, \mathrm{Ar}), 3.91-3.82(\mathrm{~m}, 3 \mathrm{H}$, $\left.\mathrm{CH}_{2}-\mathrm{N}, \mathrm{CH}-\mathrm{N}\right), 3.60-3.41\left(\mathrm{~m}, 2 \mathrm{H}, \mathrm{CH}_{2}-\mathrm{O}\right), 2.91-2.79(\mathrm{~m}, 2 \mathrm{H}$, $\mathrm{CH}_{2}-\mathrm{Ar}$ ), 2.48 (s, 3H, $\mathrm{CH}_{3}$ ) ppm. ${ }^{13} \mathrm{C} \mathrm{NMR} \mathrm{(62.90} \mathrm{MHz}, \mathrm{CDCl}_{3}$, $298 \mathrm{~K}): \delta=159.72$ (C5, pyridine); 157.98 (C1, pyridine); 141.69 (C9, phenyl); 136.45 (C3, pyridine); 129.29 (C10, phenyl); 128.89 (C11, phenyl); 126.38 (C12, phenyl); 121.33 (C4, pyridine); 119.39 (C2, pyridine); $67.62\left(\mathrm{CH}_{2}, \mathrm{CH}_{2} \mathrm{OH}\right) ; 65.10(\mathrm{CH}, \mathrm{CHNH})$; $52.89\left(\mathrm{CH}_{2}, \mathrm{CH}_{2} \mathrm{NH}\right) ; 23.97\left(\mathrm{CH}_{3}\right) \mathrm{ppm}$.

\subsubsection{Preparation of iridium complexes}

4-Methyl-2-(6-methyl-pyridin-2-yl-amino)-pent-1-oxy-iridium(I)cyclooctadiene $(\mathbf{1 a})$. [\{IrOMe $\left.(\operatorname{cod})\}_{2}\right] 0.662 \mathrm{~g}(1.00 \mathrm{mmol})$ was dissolved in THF $(20 \mathrm{~mL})$ and a solution of $0.416 \mathrm{~g}(2.00 \mathrm{mmol})$ aminopyridinato ligands (a) dissolved in THF (5 mL) was subsequently added dropwise. A red solution was obtained after addition. The reaction solution was stirred overnight at room temperature. The resulting solution was removed in vacuo and the residue was recrystallised from toluene, yielding red crystals at $-30{ }^{\circ} \mathrm{C}$, which were suitable for X-ray crystal structure analyses. Yield: $0.52 \mathrm{~g}(65 \%) . \mathrm{C}_{28} \mathrm{H}_{42} \mathrm{Ir}_{2} \mathrm{~N}_{2} \mathrm{O}$ (807.04): calcd $\mathrm{C}=41.67 ; \mathrm{H}=5.25 ; \mathrm{N}=3.47$; found $\mathrm{C}=41.92 ; \mathrm{H}=5.16$; $\mathrm{N}=3.86 .{ }^{1} \mathrm{H}$ NMR $\left(250 \mathrm{MHz}, \mathrm{C}_{6} \mathrm{D}_{6}, 296 \mathrm{~K}\right): \delta=7.43(\mathrm{t}, 1 \mathrm{H}) ; 6.34$ (dd, 2H, Ar); 4.45-4.36 (m, 4H, COD); 3.80-3.38 (m, 6H, $\mathrm{CH}_{2}-$ $\mathrm{OH}, \mathrm{COD}) ; 2.66-2.58$ (m, 1H, CH-NH); 2.54-2.27 (m, 6H, CH$\left.\mathrm{CH}_{2}-\mathrm{CH}, \mathrm{COD}\right) ; 2.07$ (s, 3H, $\left.\mathrm{CH}_{3}\right), 1.75-1.39(\mathrm{~m}, 5 \mathrm{H}, \mathrm{CH}-$ $\left.\left(\mathrm{CH}_{3}\right)_{2} / \mathrm{CH}_{2}, \mathrm{COD}\right) ; 0.96-0.78\left(\mathrm{dd}, 6 \mathrm{H}, \mathrm{CH}-\left(\mathrm{CH}_{3}\right)_{2}\right) \mathrm{ppm} .{ }^{13} \mathrm{C}$
(250 MHz, $\left.\mathrm{C}_{6} \mathrm{D}_{6}\right): 158.29,157.02,139.0,114.96,106.69\left(\mathrm{C}_{5} \mathrm{H}_{3} \mathrm{~N}\right)$; $71.47\left(\mathrm{CH}_{2}, \mathrm{CH}_{2} \mathrm{OH}\right), 68.71,64.63,62.41,60.28,60.03$ (COD); $53.95(\mathrm{CH}, \mathrm{CHNH}) ; 41.77\left(\mathrm{CH}_{2}, \mathrm{CH}_{2} \mathrm{CH}\left(\mathrm{CH}_{3}\right)_{2}\right)$; 41.56, 35.72, $34.07,33.94,32.82,32.25,32.06,31.96,30.57,30.28,25.42$ (COD); $24.88\left(\mathrm{CH}-\left(\mathrm{CH}_{3}\right)_{2}\right) ; 23.60,23.42,\left(\mathrm{CH}-\left(\mathrm{CH}_{3}\right)_{2}\right) ; 22.52$ $\left(\mathrm{CH}_{3}\right)$ ppm.

4-Methyl-2-(pyridin-2-yl-amino)-pent-1-oxy-iridium(I)-cyclooctadiene (1b). Complex 1b was prepared using $0.388 \mathrm{~g}$ ( $2.00 \mathrm{mmol}$ ) aminopyridinato ligand (b), following the above procedure for 1a. Red crystals were obtained in hexane at room temperature. Yield: $0.50 \mathrm{~g}$ (63\%). $\mathrm{C}_{27} \mathrm{H}_{40} \mathrm{Ir}_{2} \mathrm{~N}_{2} \mathrm{O}$ (793.01): calcd $\mathrm{C}=40.89 ; \mathrm{H}=5.08 ; \mathrm{N}=3.53$; found $\mathrm{C}=41.12 ; \mathrm{H}=4.98 ; \mathrm{N}=3.90 .{ }^{1} \mathrm{H} \mathrm{NMR}\left(250 \mathrm{MHz}, \mathrm{C}_{6} \mathrm{D}_{6}, 296\right.$ $\mathrm{K}): \delta=7.06(\mathrm{t}, 1 \mathrm{H}) ; 6.27$ (dd, 2H, Ar); 4.50-4.34 (m, 4H, COD); 3.843.46 (m, 6H, $\left.\mathrm{CH}_{2}-\mathrm{OH}, \mathrm{COD}\right) ; 2.77-2.70$ (m, 1H, CH-NH); 2.57-2.26 (m, 6H, CH- $\left.\mathrm{CH}_{2}-\mathrm{CH}, \mathrm{COD}\right) ; 1.49-1.25$ (m, 5H, $\left.\mathrm{CH}-\left(\mathrm{CH}_{3}\right)_{2}, \mathrm{COD}\right)$; 0.81-0.71 (dd, 6H, CH- $\left.\left(\mathrm{CH}_{3}\right)_{2}\right)$ ppm. ${ }^{13} \mathrm{C}\left(250 \mathrm{MHz}, \mathrm{C}_{6} \mathrm{D}_{6}\right): 159.23$, 148.12, 139.79, 115.96, $108.40\left(\mathrm{C}_{5} \mathrm{H}_{4} \mathrm{~N}\right)$; $76.16\left(\mathrm{CH}_{2}, \mathrm{CH}_{2} \mathrm{OH}\right), 66.42$, 66.12, 64.86, 64.56, 55.33 (COD); 51.17 (CH, CHNH); $44.99\left(\mathrm{CH}_{2}\right.$, $\left.\mathrm{CH}_{2} \mathrm{CH}\left(\mathrm{CH}_{3}\right)_{2}\right)$; 42.09, 41.24, 35.58, 34.34, 33.96, 32.41, 30.99, 28.09, 27.20, 25.19 (COD); $24.55\left(\mathrm{CH}-\left(\mathrm{CH}_{3}\right)_{2}\right) ; 23.17,22.63,\left(\mathrm{CH}-\left(\mathrm{CH}_{3}\right)_{2}\right)$ ppm.

3-Phenyl-2-[(pyridin-2-yl-methyl)-amino]-prop-1-oxy-iridium(I)cyclooctadiene (2c). To an yellow solution of $0.484 \mathrm{~g}(2.00$ mmol) pyridylalkylamine ligand (c) in $20 \mathrm{~mL}$ of THF, $0.662 \mathrm{~g}$ $(1.00 \mathrm{mmol})\left[\{\mathrm{IrOMe}(\mathrm{cod})\}_{2}\right]$ dimer was added, accompanied by a color change to dark red. The reaction solution was stirred at room temperature for $16 \mathrm{~h}$. The resulting solution was filtered and the filtrate concentrated to dryness under vacuum. The remaining solid was washed with $15 \mathrm{~mL}$ hexane and subsequently re-crystallized from toluene. Yellow crystals were grown at $-30{ }^{\circ} \mathrm{C}$, which were suitable for X-ray crystal structure analyses. Yield: $0.64 \mathrm{~g}(68 \%) . \mathrm{C}_{38} \mathrm{H}_{48} \mathrm{Ir}_{2} \mathrm{~N}_{2} \mathrm{O}$ (933.18): calcd $\mathrm{C}=$ 48.91; $\mathrm{H}=5.18 ; \mathrm{N}=3.00$; found $\mathrm{C}=48.45 ; \mathrm{H}=5.26 ; \mathrm{N}=2.86$. ${ }^{1} \mathrm{H}$ NMR (250 MHz, $\left.\mathrm{C}_{6} \mathrm{D}_{6}, 296 \mathrm{~K}\right): \delta=7.90(\mathrm{~d}, 1 \mathrm{H}) ; 7.75(\mathrm{~d}, 1 \mathrm{H})$; 7.68 (d, 1H); 7.52 (d, 1H); 7.33-6.99 (m, 5H, $\left.\mathrm{C}_{6} \mathrm{H}_{5}\right) ; 5.49(\mathrm{br}, 1 \mathrm{H}$, $\mathrm{NH}$ ); 4.55-4.40 (m, 4H, COD); 3.60-3.36 (m, 4H, COD); 3.12 (s, $2 \mathrm{H}, \mathrm{CH}_{2} \mathrm{NH}$ ); 3.00 (d, $\mathrm{CH}, \mathrm{CH}_{2} \mathrm{O}$ ); 2.69-2.62 (m, CH, CHNH); 2.27-2.16 (m, 10H, $\left.\mathrm{CH}_{2} \mathrm{C}_{6} \mathrm{H}_{5}, \mathrm{COD}\right) ; 1.64-1.48$ (m, 8H, COD) ppm. ${ }^{13} \mathrm{C}\left(250 \mathrm{MHz}, \mathrm{C}_{6} \mathrm{D}_{6}\right): \delta=165.16,147.14,135.79,134.99$, $129.59\left(\mathrm{C}_{5} \mathrm{H}_{3} \mathrm{~N}\right) ; 129.34,126.30,124.78,122.50\left(\mathrm{C}_{6} \mathrm{H}_{5}\right) ; 68.08$ $\left(\mathrm{CH},-\mathrm{CH}_{2} \mathrm{O}\right) ; 67.18(\mathrm{CH}, \mathrm{CHN}) ; 66.24,65.16,61.06,59.86$ (COD); $55.0\left(\mathrm{CH}_{2}, \mathrm{CH}_{2} \mathrm{NH}\right)$; 40.73, 35.67, 33.05, 32.14, 31.68, 31.29, 31.03, 30.74, 30.70, 28.14, 28.74, 22.76 (COD) ppm.

2-[(Pyridin-2-yl-methyl)-amino]-prop-1-oxy-iridium(I)-cyclooctadiene (2d). Complex $2 \mathrm{~d}$ was prepared using $0.332 \mathrm{~g}$ (2.00 $\mathrm{mmol}$ ) pyridylalkylamine ligand (d), following the above procedure for $2 \mathrm{c}$. At $-30{ }^{\circ} \mathrm{C}$ red crystals were obtained. Yield: $0.49 \mathrm{~g}(57 \%) . \mathrm{C}_{32} \mathrm{H}_{44} \mathrm{Ir}_{2} \mathrm{~N}_{2} \mathrm{O}$ (857.09): calcd $\mathrm{C}=44.84 ; \mathrm{H}=5.17$; $\mathrm{N}=3.27$; found $\mathrm{C}=44.80 ; \mathrm{H}=4.96 ; \mathrm{N}=3.01 .{ }^{1} \mathrm{H}$ NMR $(250$ $\left.\mathrm{MHz}, \mathrm{C}_{6} \mathrm{D}_{6}, 296 \mathrm{~K}\right): \delta=7.82(\mathrm{~d}, 1 \mathrm{H}) ; 7.55(\mathrm{~d}, 1 \mathrm{H}) ; 7.27(\mathrm{~d}, 1 \mathrm{H})$; 7.25 (d, 1H); 5.25 (br, 1H, NH); 4.42-4.29 (m, 4H, COD); 4.204.14 (m, 4H, COD); 3.94-3.90 (m, 2H, $\left.\mathrm{CH}_{2} \mathrm{NH}\right) ; 3.40$ (d, CH, $\mathrm{CH}_{2} \mathrm{O}$ ); 2.91-2.88 (m, CH, CHNH); 2.05-1.93 (m, 8H, COD); 1.54-1.34 (m, 8H, COD); 0.89 (d, 3H, $\left.\mathrm{CH}_{3}\right) \mathrm{ppm} .{ }^{13} \mathrm{C}(250 \mathrm{MHz}$, $\left.\mathrm{C}_{6} \mathrm{D}_{6}\right): \delta=165.56,147.39,140.72,124.91,122.62\left(\mathrm{C}_{5} \mathrm{H}_{4} \mathrm{~N}\right) ; 76.91$, 68.02, 67.57 (COD); $66.82\left(\mathrm{CH}_{2}, \mathrm{CH}_{2} \mathrm{OH}\right) ; 54.34$ (CH, CHNH); $51.33\left(\mathrm{CH}_{2}, \mathrm{CH}_{2} \mathrm{NH}\right)$; 49.0, 31.62, 30.87, 30.70, 30.55, 30.12, 
29.82, 28.55, 28.14, 28.02, 25.71, 23.21, 22.70 (COD); $16.16\left(\mathrm{CH}_{3}\right.$, $\left.\mathrm{CH}_{3} \mathrm{CH}\right) \mathrm{ppm}$.

2-[(6-Methyl-pyridin-2-yl-methyl)-amino]-3-phenyl-prop-1-oxyiridium(I)-cyclooctadiene (2e). Complex 2e was prepared using $0.512 \mathrm{~g}(2.00 \mathrm{mmol})$ pyridylalkylamine ligand (e) following the above procedure for 2c. Yellow crystals were grown at room temperature, which were suitable for X-ray crystal structure analyses. Yield: $0.38 \mathrm{~g}(69 \%) \cdot \mathrm{C}_{24} \mathrm{H}_{31} \mathrm{IrN}_{2} \mathrm{O}$ (555.71): calcd $\mathrm{C}=$ $51.86 ; \mathrm{H}=6.12 ; \mathrm{N}=5.13$; found $\mathrm{C}=51.96 ; \mathrm{H}=6.16 ; \mathrm{N}=5.17$. ${ }^{1} \mathrm{H}$ NMR (250 MHz, $\left.\mathrm{C}_{6} \mathrm{D}_{6}, 296 \mathrm{~K}\right): \delta=7.34(\mathrm{~d}, 1 \mathrm{H}) ; 7.27(\mathrm{~d}, 1 \mathrm{H})$; 7.23 (d, 1H); 7.20-6.35 (m, 5H, $\mathrm{C}_{6} \mathrm{H}_{5}$ ); 4.16-4.15 (m, 1H, COD); 4.09-4.04 (m, 2H, COD); 3.91-3.90 (m, 1H, COD); 3.88 (s, 2H, $\mathrm{CH}_{2} \mathrm{NH}$ ); 3.61 (d, CH, $\mathrm{CH}_{2} \mathrm{O}$ ); 3.52-3.46 (m, CH, CHNH); 2.94 (d, $\left.\mathrm{CH}, \mathrm{CH}_{2} \mathrm{C}_{6} \mathrm{H}_{5}\right) ; 2.52$ (s, 3H, $\mathrm{CH}_{3}$ ), 2.39-2.27 (m, 4H, COD); 2.23-1.89 (m, 4H, COD) ppm. ${ }^{13} \mathrm{C}\left(250 \mathrm{MHz}, \mathrm{C}_{6} \mathrm{D}_{6}\right): \delta=158.8$, $155.95,138.79,136.57,130.00\left(\mathrm{C}_{5} \mathrm{H}_{3} \mathrm{~N}\right) ; 129.28,126.91$, 122.37, $118.50\left(\mathrm{C}_{6} \mathrm{H}_{5}\right) ; 74.2\left(\mathrm{CH},-\mathrm{CH}_{2} \mathrm{O}\right) ; 70.5(\mathrm{CH}, \mathrm{CHN})$; 61.38, 58.03 (CH, COD); $55.9\left(\mathrm{CH}_{2}, \mathrm{CH}_{2} \mathrm{NH}\right) ; 52.07,51.7(\mathrm{CH}$, COD); $38.9\left(\mathrm{CH}, \mathrm{CH}_{2}-\mathrm{C}_{6} \mathrm{H}_{5}\right)$; 33.85, 32.44, 31.35, $31.14\left(\mathrm{CH}_{2}\right.$, COD); $25.5\left(\mathrm{CH}_{3}\right)$ ppm.

Complexes $\mathbf{2 f - h}$ : not isolated, pre-catalyst-solution prepared via alcohol elimination in situ.

2.2.4. General procedure for the asymmetric hydrogenation. All catalytic hydrogenation experiments were carried out in stainless steel autoclaves (Parr instrument N-MT5 $300 \mathrm{~mL}$ ) and in a mmol scale. All experiments were carried out in a glove box under exclusion of oxygen and moisture. Stock solutions of the pre-catalysts were prepared in THF via alcohol elimination reaction of the ligand (stock solution in THF) and 0.5 equiv. of $\left[\mathrm{IrOCH}_{3}(\mathrm{cod})\right]_{2}$. The solutions were prepared and stored in a glove box. A high pressure steel autoclave (Parr Instruments; $300 \mathrm{~mL}, 200 \mathrm{bar}, 350{ }^{\circ} \mathrm{C}$ ) with an aluminium insert for multiple reaction tubes ( 5 or 20 ) was taken into a glove box. Then the reaction tube (placed in a 20- or 5-well insert for the autoclave, equipped with a magnetic stir bar) was loaded with additive (base if required), the pre-catalyst solution (e.g. $100 \mu \mathrm{L}=0.05$ $\mathrm{mol} \%)$ and $350 \mu \mathrm{L}(2.29 \mathrm{mmol})$ of the substrate solution. Then the autoclave was sealed and taken out of the glove box. The autoclave was attached to a high-pressure hydrogen line and purged with $\mathrm{H}_{2}$. The autoclave was sealed under the appropriate $\mathrm{H}_{2}$ pressure and the mixture was stirred for e.g. $48 \mathrm{~h}$ at the appropriate pressure at room temperature or at the appropriate temperature (external heating mantle). In order to stop the hydrogenation reaction, the pressure was released and water and dodecane (standard for GC) were added to the reaction.

2.2.5. Single crystal X-ray structure determinations. Crystals suitable for single crystal X-ray diffraction analyses for 1a, 1b, 2c, 2d and 2e contained toluene as a solvate. Preliminary data on the space group and unit cell dimensions as well as intensity data were collected by using a STOE-IPDS II equipped with an Oxford Cryostream low temperature unit using graphite monochromatized Mo-K $\alpha$ radiation. Structure solution and refinement were accomplished using SIR97, ${ }^{18}$ SHELXL-97 (ref. 19) and WinGX. ${ }^{20}$ The non-hydrogen atoms were refined with anisotropic thermal parameters. Hydrogen atoms were geometrically fixed and allowed to refine using riding model.
CCDC deposition numbers 1437480-1437485 contain supplementary crystallographic data in CIF format for this paper. $\dagger$

\section{Results and discussion}

\subsection{Ligand synthesis}

The targeted ligands were obtained from commercially available reactants in one or two steps based on previous studies. ${ }^{21}$ The ligand precursors 4-methyl-2-(6-methyl-pyridin-2-yl-amino)pentan-1-ol (a) and 4-methyl-2-(pyridin-2-yl-amino)-pentan-1-ol (b) were synthesized in a one-pot reaction starting from 6methyl 2-bromopyridines and 2-bromopyridines with enantiopure amino alcohols using $N, N$-diisopropylethyl amine as a base at $165{ }^{\circ} \mathrm{C}$ for 3 days (Scheme 2) in quantitative yields. ${ }^{22}$ Ligands $(\mathbf{c}-\mathbf{h})$ were prepared by reductive amination of heteroaromatic aldehydes (pyridine-2-carbaldehyde or 6-methylpyridine-2carbaldehyde) with enantiopure aminoalcohols over two steps including the formation of the corresponding ketimine and further reduction with sodium borohydride (Scheme 3). Both methodologies allowed us to prepare a family of substituted ligands based on a pyridylalkylamine and aminopyridinato core. All the synthesized ligands are air stable and soluble in polar as well as non-polar solvents and characterized by ${ }^{1} \mathrm{H},{ }^{13} \mathrm{C}$ NMR and elemental analysis.

\subsection{Synthesis and structure of the complexes}

The dinuclear iridium complexes $\mathbf{1 a}, \mathbf{b}$ and $\mathbf{2 c}$, $\mathbf{d}$ can be obtained via alcohol elimination route with $\left[\mathrm{IrOCH}_{3}(\mathrm{cod})\right]_{2}$ (ref. 23) (Scheme 2 and 3). Addition of 0.5 equiv. of the metal precursor to a solution of $\mathbf{a}-\mathbf{d}$ in THF gives rise to $\mathbf{1 a}, \mathbf{b}$ and $\mathbf{2 c}$, d which is accompanied by a color change to yellow/reddishbrown. While under the same reaction condition mononuclear iridium complexes $\mathbf{2} \mathbf{e}-\mathbf{h}$ can be synthesized by reaction with $\left[\mathrm{IrOCH}_{3}(\mathrm{cod})\right]_{2}$ (Scheme 3 ). This synthetic approach shows the presence of the methyl group, at sixth position of pyridine ring, and the methylene $\left(-\mathrm{CH}_{2} \mathrm{NH}\right)$ group, favour the formation of mononuclear iridium complex.

X-ray crystal structure analyses of the dinuclear complexes (1a, b and 2c, d) and the mononuclear (2e) were performed to determine the molecular structures (Fig. 1-5). Selected crystallographic data and geometrical parameters are summarized in the Fig. 1-5, respectively and details about the data collection, solution, and refinement are summarized in the ESI (Table S1 $\dagger$ ). In complexes $\mathbf{1 a}$ and $\mathbf{1 b}$ (Fig. 1 and 2) the Ir1-atoms are stabilized by the formation of a five membered chelate (Ir1, N1, C9, C10, O1) and Ir2-atom is stabilized by the formation of a four membered chelate (Ir2, N1, N2, C15). It was observed that Ir- $\mathrm{N}_{\text {amido }}$ bond

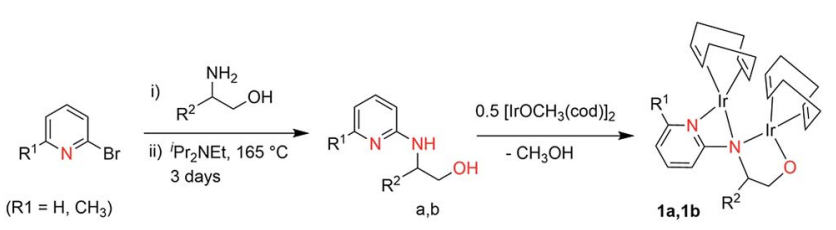

Scheme 2 Synthesis of iridium complexes 1 (a) $\mathrm{R}^{1}=\mathrm{CH}_{3}, \mathrm{R}^{2}=\mathrm{CH}_{2}-$ $\left(\mathrm{CH}_{3}\right)_{2}$, (b) $\mathrm{R}^{1}=\mathrm{H}, \mathrm{R}^{2}=\mathrm{CH}_{2}-\mathrm{CH}\left(\mathrm{CH}_{3}\right)_{2}$. 


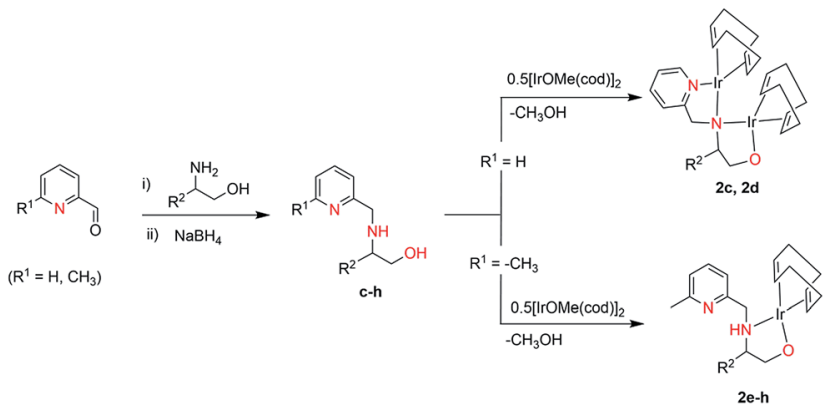

Scheme 3 Synthesis of iridium complexes 2 (c) $\mathrm{R}^{1}=\mathrm{H}, \mathrm{R}^{2}=\mathrm{CH}_{2}-$ $\mathrm{C}_{6} \mathrm{H}_{5}$, (d) $\mathrm{R}^{1}=\mathrm{H}, \mathrm{R}^{2}=\mathrm{CH}_{3}$, (e) $\mathrm{R}^{1}=\mathrm{CH}_{3}, \mathrm{R}^{2}=\mathrm{CH}_{2}-\mathrm{C}_{6} \mathrm{H}_{5}$, (f) $\mathrm{R}^{1}=\mathrm{CH}_{3}$, $\mathrm{R}^{2}=\mathrm{CH}_{2}-\mathrm{CH}\left(\mathrm{CH}_{3}\right)_{2}$, (g) $\mathrm{R}^{1}=\mathrm{CH}_{3}, \mathrm{R}^{2}=\mathrm{CH}_{3}$, (h) $\mathrm{R}^{1}=\mathrm{CH}_{3}, \mathrm{R}^{2}=\mathrm{C}_{6} \mathrm{H}_{5}$.

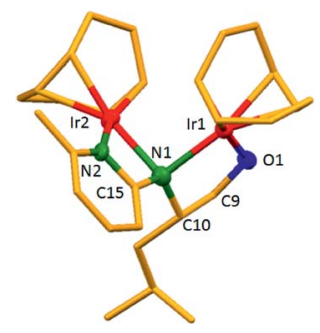

Fig. 1 Molecular structure of 1a. [Thermal ellipsoid diagram (50\%) for all non-carbon atoms] without $\mathrm{H}$ atoms. Selected bond length $[\AA \AA]$ and angles [ ${ }^{\circ}$ ]: Ir1-O1 1.979(8), Ir1-N1 2.144(10), Ir2-N1 2.145(10), Ir2-N2 2.020(10), O1-C9 1.413(15), N1-C10 1.490(15), N1-C15 1.388(15); N1Ir1-O1 80.6(4), N1-Ir2-N2 63.5(4), Ir2-N1-Ir1 94.0(4), Ir1-O1-C9 114.9(7), C10-N1-Ir2 130.7(7), C10-N1-Ir1 106.0(7).

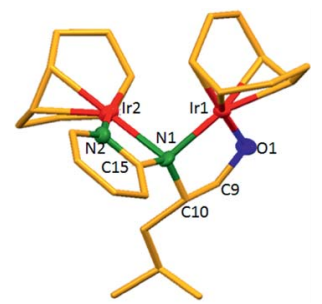

Fig. 2 Molecular structure of 1b. [Thermal ellipsoid diagram for all non-carbon atoms] without $\mathrm{H}$ atoms. Selected bond length $[\AA \AA]$ and angles [ ${ }^{\circ}$ ]: Ir1-O1 1.998(5), Ir1-N1 2.086(6), Ir2-N1 2.202(5), Ir2-N2 2.062(6), O1-C9 1.401(9), N1-C10 1.491(9), N1-C15 1.414(11); N1-Ir1O1 80.3(2), N1-Ir2-N2 63.5(2), Ir2-N1-|r1 82.73(16), Ir1-O1-C9 115.2(5), C10-N1-Ir2 108.1(14), 128.4(4), C10-N1-Ir1 109.9(4).

[Ir2-N1 2.145(10) 1a, Ir2-N1 2.202(5), §] is significantly longer than Ir- $\mathrm{N}_{\text {pyridine }}$ bond [Ir2-N2 2.020(10) 1a, N2-Ir2 2.062(6), Å 1b]. These simply suggest that the anionic charge of the ligand is not localized at the amido-N-atom. The Ir-O bond is significantly shorter than the Ir-N bond indicating the localization of the negative charge of the alkoxy $\left(-\mathrm{CH}_{2} \mathrm{OH}\right)$ at the oxygen atom. The bite angle N1-Ir1-O1 $\left(80.6^{\circ}\right)$ is almost equal to $2 \mathrm{c}$, but significantly smaller than $2 \mathbf{e}$. While in $\mathbf{2 c}$ and $\mathbf{2 d}$, the two iridium atoms (Ir1 and Ir2) are stabilized by the formation of a five membered chelate (Ir1, N2, C9, C10, O1, and Ir2, N1, N2, C19, C18 2c; Ir2, N1,
Fig. 3 Molecular structure of 2c. [Thermal ellipsoid diagram for all non-carbon atoms] without $\mathrm{H}$ atoms. Selected bond length $[\AA \AA]$ and angles [ ${ }^{\circ}$ ]: Ir1-O1 2.021(6), Ir1-N2 2.151(6), Ir2-N1 2.103(7), Ir2-N2 2.106(6), O1-C9 1.356(9), N2-C10 1.452(10); N2-Ir1-O1 80.9(2), N1Ir2-N2 79.7(2), Ir2-N2-Ir1 89.7(2), Ir1-O1-C9 112.4(5), C10-N2-Ir2 119.0(5), C18-N2-Ir1 119.1(5), C18-N2-Ir2 109.8(5), C10-N2-Ir1 107.1(5), N2-C18-C19 112.2(6).

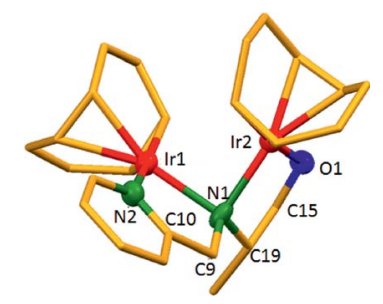

Fig. 4 Molecular structure of $\mathbf{2 d}$. [Thermal ellipsoid diagram for all non-carbon atoms] without $\mathrm{H}$ atoms. Selected bond length $[\AA \AA]$ and angles [ ${ }^{\circ}$ ]: Ir2-O1 2.017(12), Ir1-N1 2.145(14), Ir2-N1 2.150(18), Ir1-N2 2.089(16), O1-C16 1.39(2), N1-C15 1.47(3); N1-Ir2-O1 80.6(6), N1Ir1-N2 80.6(7), Ir2-N1-Ir1 88.7(6), Ir2-O1-C16 114.4(11), C15-N1-Ir2 108.1(14), C15-N1-Ir1 125.6(13), C9-N1-Ir1 109.6(11), C9-N1-Ir2 118.3(13), C10-N2-Ir1 116.1(13), N1-C9-C10 112.3(15).

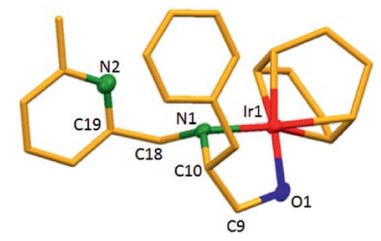

Fig. 5 Molecular structure of 2e. [Thermal ellipsoid diagram for all non-carbon atoms] without $\mathrm{H}$ atoms. Selected bond length $[\AA \AA]$ and angles [']: Ir1-O1 1.995(3), Ir1-N1 2.127(3), O1-C9 1.425(5), C9-C10 1.530(6), N1-C18 1.492(6); N1-Ir1-O1 82.67(13), C9-O1-Ir1 112.7(3), C9-C10-N1 106.1(4), C10-N1-Ir1 105.9(3), N1-C18-C19 110.4(4).

C15, C16, O1, and Ir1, N1, N2, C9, C10 2d). Again, the Ir-O bond [2.021(6) $\mathrm{A}]$ is significantly shorter than the $\mathrm{Ir}-\mathrm{N}$ bond [2.151(6) $\mathrm{A}]$ and Ir-N bond [2.103(7) $\AA]$ and Ir-N bond [2.106(6) $\AA]$ are almost equal in 2c and $2 d$ (Fig. 3 and 4 ).

Furthermore, in complexes 2e (Fig. 5) the monoanionic ligand coordinates the Ir-atom via a five membered chelate (Ir1, N1, C9, C10, and O1). The Ir1-O1 bond [1.995(3) ̊] is significantly shorter than the Ir1-N1 bond [2.127(3) A] , simply indicating the localization of the anionic charge of the alkoxy at the oxygen atom. The 6-methylpyridyl moiety is bent out of the pyridine plane (N1-C18-C19 $110.4^{\circ}$ ) and coordination by the pyridine nitrogen does not occur. However, the bite angle of 
Table 1 Asymmetric hydrogenation of ( $\alpha$-methylpropiophenone) with $2 \mathrm{e}-\mathrm{h}^{a}$

\begin{tabular}{llllc}
\hline Entry & Pre-catalyst & Base & Conv. $^{b}(\%)$ & $\mathrm{ee}^{b}(\%)$ \\
\hline 1 & $2 \mathbf{e}$ & - & 55 & 10 \\
2 & $\mathbf{2 e}$ & $\mathrm{KO}^{t} \mathrm{Bu}$ & $>99$ & 89 \\
3 & $\mathbf{2 f}$ & - & 46 & 8 \\
4 & $\mathbf{2 f}$ & $\mathrm{KO}^{t} \mathrm{Bu}$ & $>99$ & 74 \\
5 & $\mathbf{2 g}$ & - & 16 & 5 \\
6 & $\mathbf{2 g}$ & $\mathrm{KO}^{t} \mathrm{Bu}$ & 64 & 82 \\
7 & $\mathbf{2 h}$ & - & 28 & 10 \\
8 & $\mathbf{2 h}$ & $\mathrm{KO}^{t} \mathrm{Bu}$ & 55 & 68
\end{tabular}

${ }^{a}$ Reaction conditions: $0.05 \mathrm{~mol} \% \mathbf{2 e - h}, \mathrm{THF}, \mathrm{KO}^{t} \mathrm{Bu} / 2 \mathbf{e}-\mathbf{h} 500: 1,48 \mathrm{~h}$, $20{ }^{\circ} \mathrm{C}, 20$ bar $\mathrm{H}_{2} .{ }^{b}$ Determined via GC.

complex 1a (N1-Ir1-O1, 80.6 $)$, 1b (N1-Ir1-O1, 80.3), 2c (N2Ir1-O1, 80.9 $9^{\circ}$, and 2d (N1-Ir1-O1, 80.6 $)$ are almost same but slightly smaller than $2 \mathrm{e}\left(\mathrm{N} 1-\mathrm{Ir} 1-\mathrm{O} 1,82.67^{\circ}\right)$. The increased angle brought the Ir atom, the reaction center of the catalyst, closer to the ligand backbone and created a chiral environment around the Ir atom that permitted more efficient catalysis.

\subsection{Catalytic studies}

Based on the pioneering work of Kempe et al. group ${ }^{24}$ where amido iridium complexes were employed as a catalyst for the asymmetric hydrogenation of the simple ketones, we have chosen to determine the catalytic potential of iridium $-\mathrm{N}^{\wedge} \mathrm{N}^{\wedge} \mathrm{O}$ complexes for such reactions. In general, the screening reactions were performed using $2.29 \mathrm{mmol}$ of the substrate at $20{ }^{\circ} \mathrm{C}$ for $48 \mathrm{~h}$ and the catalyst was prepared in situ from stock solutions of $\left[\mathrm{IrOCH}_{3}(\mathrm{cod})\right]_{2}$ and $\mathrm{N}^{\wedge} \mathrm{N}^{\wedge} \mathrm{O}$ ligand.

First of all, mononuclear iridium complexes $\mathbf{2 e - h}$ (Scheme 3) were used to determine the effect of substitution at the amino skeleton of the ligands on the asymmetric hydrogenation of simple ketones (Table 3). Evidently, the catalysts $2 \mathbf{e}$ and $\mathbf{2 f}$ containing benzyl and isopropyl substituents on the nitrogen (Table 1, entries 2 and 4) gave better results than those containing the methyl (2g) or phenyl (2h) substituent (Table 1, entries 6 and 8). The best catalyst for this reaction seems to be compound 2e (Table 1, entry 2), which achieved a $>99 \%$ conversion and $89 \%$ ee at a very low catalyst loading (0.05 mol\% iridium) and excess $\mathrm{KO}^{t} \mathrm{Bu}$.

In this catalytic work, all optimization studies were carried out with $\alpha$-methyl propiophenone as model substrates. As can

Table 2 Base screening, base/pre-catalyst $500: 1,48$ h, 0.05 mol\% 2e, $20{ }^{\circ} \mathrm{C}$, and $\mathrm{H}_{2}$ (20 bar)

\begin{tabular}{llll}
\hline Entry & Base & Conv. [\%] & ee [\%] \\
\hline 1 & - & 55 & 10 \\
2 & $\mathrm{NaNH}_{2}$ & 25 & 65 \\
3 & $\mathrm{KOSiMe}_{3}$ & 40 & 34 \\
4 & $\mathrm{KOH}$ & 64 & 30 \\
5 & $\mathrm{NaO}^{t} \mathrm{Bu}$ & 46 & 74 \\
6 & $\mathrm{KO}^{t} \mathrm{Bu}$ & $>99$ & 89 \\
7 & $\mathrm{KN}\left(\mathrm{SiMe}_{3}\right)_{2}$ & 69 & 80
\end{tabular}

be seen from Table $2, \mathrm{KO}^{t} \mathrm{Bu}$ appears to be the most suitable base, because complete conversion and a good ee (89\%) could only be achieved by using this base (Table 2, entry 6 ). This result shows that $\mathrm{KO}^{t} \mathrm{Bu}$ is needed as an additive for better enantioselectivity during hydrogenation. Presence of base $\mathrm{KO}^{t} \mathrm{Bu}$ is essential for catalysis, because base itself act as a catalyst for the hydrogenation of simple ketones under drastic conditions, ${ }^{25}$ its potassium cations accelerate the reaction rate of phosphaneruthenium-diamine complexes. ${ }^{26}$

After these optimisations, we examined the addition of excess amounts of base was needed to allow complete conversion or whether catalytic quantities of base are sufficient. Therefore, the influence of the base/catalyst ratio was investigated (Table 3, entries 1-5). The results shown in Table 3, entries 1-5, suggest that it is necessary to use a base/catalyst ratio of $500: 1$, because only in this case (Table 3, entry 5) was it possible to obtain complete conversion and a better ee (96\%) within $48 \mathrm{~h}$ in the presence of catalytic amount of acetone (acetone : $\alpha$-methylpropiophenone $2: 1$ ).

However, at a base loading of only $5 \mathrm{~mol} \%$, it was possible to achieve a conversion (>99\%) and ee (90\%) (Table 3, entry 4 ), which contradicts the aforementioned excess requirement for base. For this reason, we investigated whether it is possible to bring the reaction to complete conversion or better ee by the use of $25 \mathrm{~mol} \%$ of $\mathrm{KO}^{t} \mathrm{Bu}$. To this end, the reaction time was $48 \mathrm{~h}$ an ee of $96 \%$ were obtained.

The final screening was performed on the catalyst loading to find the minimum catalyst loading necessary to achieve full conversion and good ee (Fig. 6). As shown in Fig. 6, it was sufficient to use a catalyst loading of $0.05 \mathrm{~mol} \%$ to obtain a very good ee (96\%) for this reaction (Fig. 6). It was observed the addition of $\mathrm{KO}^{t} \mathrm{Bu}$ and acetone leads to quantitative conversion with $2 \mathrm{e}$ as a pre-catalyst with catalyst loadings of 1, 0.5, 0.2, 0.1, and $0.05 \mathrm{~mol} \%$ (Fig. 6). It was examined that without $\mathrm{KO}^{t} \mathrm{Bu}$ and acetone with $1.0 \mathrm{~mol} \%$ of $2 \mathrm{e}$ a conversion of $55 \%$ and $10 \%$ ee is obtained (Fig. 6, Table 1, and entry 1). The catalytic performance for hydrogenation of $\boldsymbol{\alpha}$-methylpropiophenone of $\mathbf{2 e - h}$ with $\mathrm{KO}^{t} \mathrm{Bu}$ and acetone was examined (Table 4). Upon addition of acetone in the presence of $\mathrm{KO}^{t} \mathrm{Bu}, 96 \%$ ee could be achieved under same reaction condition with $0.05 \mathrm{~mol} \%$ catalyst loading (Table 4, entry 2). The increase in enantioselectivity could result from the fact that the more enantioselective catalyst species is formed during catalysis, the addition of (non-prochiral) ketones, which is hydrogenated in parallel and preferentially faster, should support the formation of a more enantioselective catalyst.

Table 3 Screening - amount of $\mathrm{KO}^{t} \mathrm{Bu}$, acetone : $\alpha$-methyl-propiophenone 2 : 1 , THF, $48 \mathrm{~h}, 0.05 \mathrm{~mol} \% 2 \mathrm{e}, 20{ }^{\circ} \mathrm{C}, 20 \mathrm{bar}$

\begin{tabular}{llll}
\hline Entry & Base $:$ pre-catalyst & Conv. (\%) & ee (\%) \\
\hline 1 & $1: 1(0.05 \mathrm{~mol} \%)$ & 60 & 32 \\
2 & $10: 1(0.5 \mathrm{~mol} \%)$ & 78 & 60 \\
3 & $50: 1(2.5 \mathrm{~mol} \%)$ & 96 & 82 \\
4 & $100: 1(5 \mathrm{~mol} \%)$ & $>99$ & 90 \\
5 & $500: 1(25 \mathrm{~mol} \%)$ & $>99$ & 96
\end{tabular}



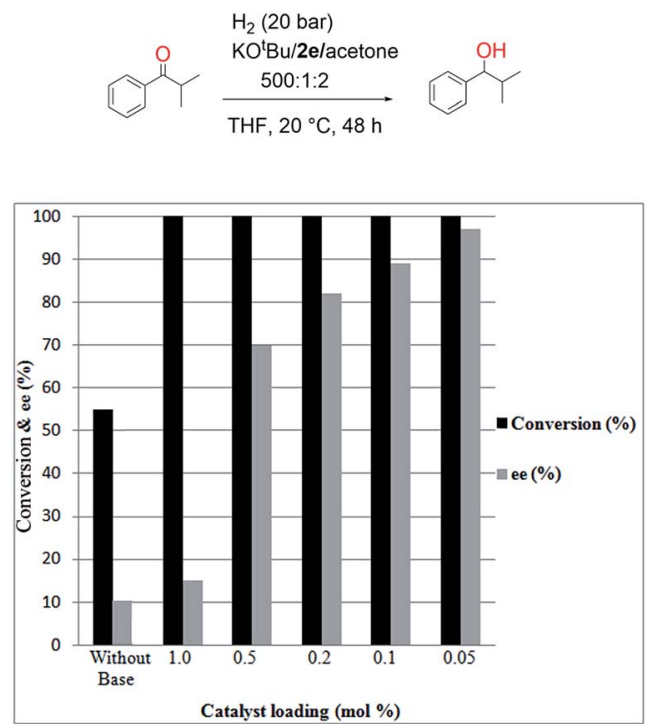

Fig. 6 Hydrogenation of $\alpha$-methylpropiophenone with $2 \mathrm{e}$ in the presence of $\mathrm{KO}^{t} \mathrm{Bu}$ and acetone.

As it can be seen in Table 4, catalyst $2 \mathrm{e}$ and $\mathbf{2 f}$ are active catalyst for asymmetric hydrogenation of $\alpha$-methylpropiophenone. Further, we were interested to examine the effect of acetone on the reaction. It was observed that acetone/ pre-catalyst 2 e ratio of $2: 1$ was use to obtain complete conversion ( $>99 \%)$ and good ee (96\%). It was also observed that if we changed the acetone/catalyst ratio (1:1 and $3: 1)$, considerably affect on the conversion ( $86 \%$ and $90 \%)$ and ee (80\% and $86 \%)$.

To confirm the results we achieved for the hydrogenation $\alpha$ methylpropiophenone with catalyst $\mathbf{2 e}$, different simple aromatic ketones were further examined (Fig. 7). These results show that the reduction of the hindered aromatic ketones proceeded with excellent enantioselectivity, although the expected trend of a decrease in conversion and enantioselectivity for the more bulky $t$-butyl substituent was also observed (Fig. 7). Also with the increase of the $\alpha$-carbon chain $\left(-\mathrm{CH}_{2} \mathrm{CH}_{2} \mathrm{CH}_{3}\right)$ (Fig. 7, VIII), the selectivity and activity of the reaction drops significantly. This result illustrates the importance of bulky

Table 4 Asymmetric hydrogenation of ( $\alpha$-methylpropiophenone) with $2 \mathrm{e}-\mathrm{h}^{a}$

\begin{tabular}{lllll}
\hline Entry & Pre-catalyst & $\begin{array}{l}\text { Acetone/ } \alpha \text {-methyl- } \\
\text { propiophenone }\end{array}$ & Conv. $^{b}(\%)$ & ee $^{b}(\%)$ \\
\hline 1 & $2 \mathbf{e}$ & - & $>99$ & 89 \\
2 & $2 \mathbf{e}$ & $2: 1$ & $>99$ & 96 \\
3 & $\mathbf{2 f}$ & - & $>99$ & 74 \\
4 & $\mathbf{2 f}$ & $2: 1$ & $>99$ & 94 \\
5 & $\mathbf{2 g}$ & - & 64 & 82 \\
6 & $\mathbf{2 g}$ & $2: 1$ & 68 & 88 \\
7 & $\mathbf{2 h}$ & - & 55 & 68 \\
8 & $\mathbf{2 h}$ & $2: 1$ & 70 & 82
\end{tabular}

${ }^{a}$ Reaction conditions: $0.05 \mathrm{~mol} \% 2 \mathrm{e}-\mathrm{h} \mathrm{KO}^{t} \mathrm{Bu} /$ pre-catalyst $500: 1,48 \mathrm{~h}$, $20{ }^{\circ} \mathrm{C}, 20{\text { bar } \mathrm{H}_{2} .}^{b}$ Determined via GC.

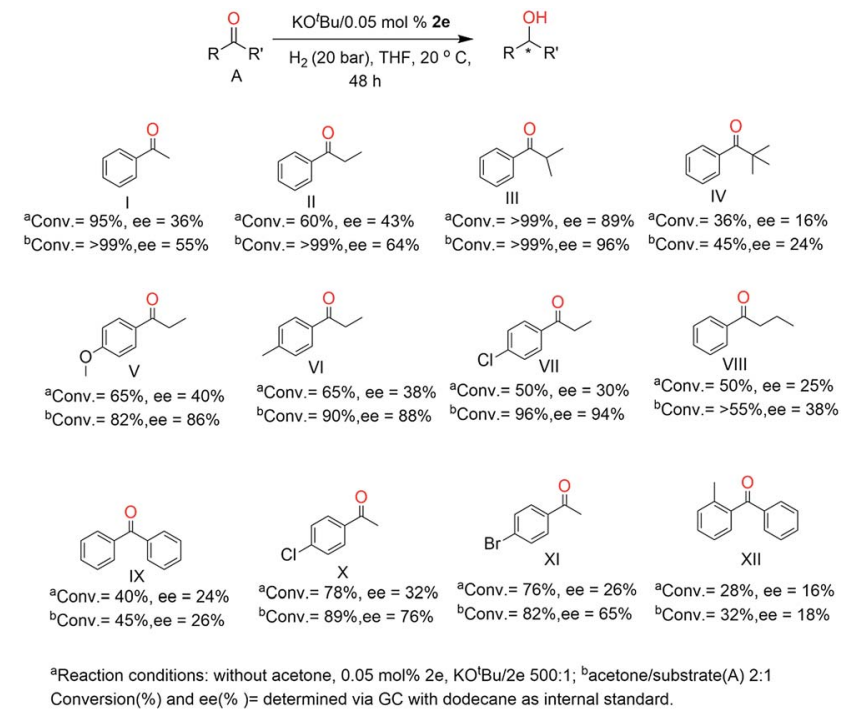

Fig. 7 Asymmetric hydrogenation of ketones catalyzed by $2 \mathrm{e}$.

groups next to the carbonyl for obtaining high enantioselectivity. The aromatic ketones (Fig. 7, VII) with a strong $\sigma$ electron withdrawing and weak $\pi$-electron donating chloro group and with the $\pi$-electron donating methoxy and methyl group in the para position showed a higher selectivity and lower activity compared to that with propiophenone. These results indicate that the para substituted ketones have major effect on the catalytic hydrogenation of ketones.

In contrast, the dinuclear iridium complexes $\mathbf{1 a}, \mathbf{b}$ and $2 \mathbf{c}$, d show lower catalytic activity and selectivity for the hydrogenation of simple ketones. For example asymmetric hydrogenation of $\alpha$-methylpropiophenone catalyzed by $1 \mathrm{a}$ and $2 \mathrm{c}(0.05$ $\mathrm{mol} \%$ ) in the presence of excess $\mathrm{KO}^{t} \mathrm{Bu}$ and acetone under same reaction condition (see Table $\mathrm{S} 2 \dagger$ ) gave corresponding alcohol in $40 \%$ and $35 \%$ yield and $24 \%$ and $8 \%$ ee. While $\mathbf{1 b}$ and $2 \mathrm{~d}$ gave $23 \%$ and $16 \%$ yield and $11 \%$ and $2.0 \%$ ee. Needless to say, no reaction occurred in the absence of $\mathrm{KO}^{t} \mathrm{Bu}$. This may suggest that dinuclear iridium complexes act as catalyst precursor to promote enantioselective asymmetric hydrogenation under the influence of base and acetone.

\section{Conclusions}

In conclusion the reported pyridylalkylamine mononuclear iridium complexes $\mathbf{2} \mathbf{e}-\mathbf{h}$ represents a novel class of efficient and easily accessible catalysts for the asymmetric hydrogenation of simple ketones. Due to the modular ligand design, broad substitution patterns can be realized. The moderate to high efficiency and good selectivity combined with the novel structural motif opens up new prospects for the enantioselective hydrogenation of ketones.

\section{Acknowledgements}

Financial support from Deutsche Forschungsgemeinschaft is gratefully acknowledged. P. Kumar is thankful to the Deutscher Akademischer Austausch Dienst (DAAD) for a fellowship. 


\section{Notes and references}

1 (a) R. M. Bullock, Chem.-Eur. J., 2004, 10, 2366; (b) R. M. Bullock, in Handbook of Homogeneous hydrogenation, ed. J. G. de Vries and C. J. Elsevier, Wiley-VCH, Weinheim, 2007, ch. 7; (c) R. Noyori and T. Ohkuma, Angew. Chem., 2001, 113, 40; (d) R. Noyori and T. Ohkuma, Angew. Chem., Int. Ed., 2001, 40, 40; (e) N. B. Johnson, I. C. Lennon, P. H. Moran and J. A. Ramsden, Acc. Chem. Res., 2007, 40, 1291.

2 W. S. Knowles, Angew. Chem., Int. Ed., 2002, 41, 1998.

3 W. S. Knowles and R. Noyori, Acc. Chem. Res., 2007, 40, 1238. 4 R. Noyori, Angew. Chem., Int. Ed., 2002, 41, 2008.

5 (a) N. B. Johnson, I. C. Lennon, P. H. Moran and J. A. Ramsden, Acc. Chem. Res., 2007, 40, 1291; (b) C. S. Schultz and S. W. Krska, Acc. Chem. Res., 2007, 40, 1320; (c) H. Shimizu, I. Nagasaki, K. Matsumura, N. Sayo and T. Saito, Acc. Chem. Res., 2007, 40, 1385.

6 (a) R. Noyori, Asymmetric Catalysis in Organic Synthesis, Wiley, New York, 1994,; (b) R. Noyori, Chem. Soc. Rev., 1989, 18, 187; (c) R. Noyori and S. Hashiguchi, Acc. Chem. Res., 1997, 30, 97.

7 (a) P. Braunstein, F. Naud and S. J. Rettig, New J. Chem., 2001, 25, 32; (b) P. Braunstein, M. D. Fryzuk, F. Naud and S. J. Rettig, J. Chem. Soc., Dalton Trans., 1999, 589; (c) P. Braunstein, F. Naud, C. Graiff and A. Tiripicchio, Chem. Commun., 2000, 897.

8 W. A. Herrmann and B. Cornils, Angew. Chem., Int. Ed., 1997, 36, 1049.

9 (a) R. H. Crabtree, H. Felkin and G. E. Morris, J. Organomet. Chem., 1977, 135, 205; (b) R. H. Crabtree and G. E. Morris, J. Organomet. Chem., 1977, 135, 395; (c) R. H. Crabtree, Acc. Chem. Res., 1979, 12, 331; (d) R. H. Crabtree, H. Felkin, T. Fillebeen-Khan and G. E. Morris, J. Organomet. Chem., 1979, 168, 183.

10 Examples of phosphorus-free ruthenium catalysts: (a) M. Ito, M. Hirakawa, K. Murata and T. Ikariya, Organometallics, 2001, 20, 379; (b) I. Karame, M. Jahjah, A. Messaoudi, M. L. Tommasino and M. Lemaire, Tetrahedron: Asymmetry, 2004, 15, 1569; (c) T. Ohkuma, N. Utsumi, K. Tsutsumi, K. Murata, C. A. Sandoval and R. Noyori, J. Am. Chem. Soc., 2006, 128, 8724; (d) C. A. Sandoval, T. Ohkuma, N. Utsumi, K. Tsutsumi, K. Murata and R. Noyori, Chem.-sian J., 2006, 1-2, 102; (e) M. L. Tommasino, C. Thomazeau, F. Touchard and M. Lemaire, Tetrahedron: Asymmetry, 1999, 10, 1813; $(f)$ M. L. Tommasino, M. Casalata, J. A. Breuzard, M. C. Bonnet and M. Lemaire, Stud. Surf. Sci. Catal., 2000, 130D, 3369; $(g)$ C. Maillet, T. Praveen, P. Janvier, S. Minguet, M. Evain, C. Saluzzo, M. L. Tommasino and B. Bujoli, J. Org. Chem., 2002, 67, 8191; (h) J. Liu, X. Wu, X. Wu, J. A. Iggo and J. Xiao, Coord. Chem. Rev., 2008, 252, 782; (i) J. E. D. Martins, D. J. Morris and M. Wills, Tetrahedron Lett., 2009, 50, 688.

11 (a) X. Wu, C. Corcoran, S. Yang and J. Xiao, ChemSusChem, 2008, 1, 7; (b) S.-I. Inuoe, K. Nomura, S. Hashiguchi, R. Noyori and Y. Izawa, Chem. Lett., 1987, 957.
12 (a) R. Noyori, Adv. Synth. Catal., 2003, 345, 15; (b) R. Noyori, C. A. Sandoval, K. Muniz and T. Ohkuma, Philos. Trans. $R$. Soc. London, Ser. A, 2005, 363, 901; (c) C. Hedberg, in Modern Reduction Methods, ed. P. G. Andersson and I. J. Munslow, Wiley-VCH, 2008, ch. 5;; (d) R. Noyori and T. Ohkuma, Angew. Chem., Int. Ed., 2006, 40, 40.

13 (a) H. Doucet, T. Ohkuma, K. Murata, T. Yokozawa, E. Katayama, A. F. England, T. Ikariya and R. Noyori, Angew. Chem., Int. Ed., 1998, 37, 1703; (b) T. Ohkuma, H. Ooka, S. Hashiguchi, T. Ikariya and R. Noyori, J. Am. Chem. Soc., 1995, 117, 2675; (c) C. A. Sandoval, T. Ohkuma, K. Muniz and R. Noyori, J. Am. Chem. Soc., 2003, 125, 13490; (d) R. Abbel, K. Abdur-Rashid, M. Faatz, A. Hadzovic, A. J. Lough and R. H. Morris, J. Am. Chem. Soc., 2005, 127, 1870; (e) T. Leysson, D. Peters and J. N. Harvey, Organometallics, 2008, 27, 1514.

14 (a) V. Rautenstrauch, X. Hoang-Cong, R. Churland, K. AbdurRashid and R. H. Morris, Chem.-Eur. J., 2003, 9, 4954; (b) J.-X. Gao, H. Zhang, X.-D. Yi, P.-P. Xu, C.-L. Tang, H.-L. Wan, K.-R. Tsai and T. Ikariya, Chirality, 2000, 12, 383. 15 (a) T. Li, R. Churland, A. J. Lough, K. Abdur-Rashid and R. H. Morris, Organometallics, 2004, 23, 6239; (b) R. J. Hamilton and S. H. Bergens, J. Am. Chem. Soc., 2006, 128, 13700; (c) S. E. Clapham, R. Guo, M. Zimmer-De Iuliis, N. Rasool, A. Lough and R. H. Morris, Organometallics, 2006, 25, 5477; (d) R. Puchta, L. Dahlenburg and T. Clark, Chem.-Eur. J., 2008, 14, 8898.

16 C. S. Chin and B. Lee, J. Chem. Soc., Dalton Trans., 1991, 1323. 17 L. A. Oro and J. A. Cabeza, Inorg. Synth., 1985, 23, 128.

18 A. Altomare, M. C. Burla, M. Camalli, G. L. Cascarano, C. Giacovazzo, A. Guagliardi, A. G. G. Moliterni, G. Polidori and R. Spagna, J. Appl. Crystallogr., 1999, 32, 115.

19 G. M. Sheldrick, SHELX-97, Programm for Cystal Structure Analysis (Release 97-2), Institut für Anorganische Chemie der Universität Göttingen, Germany, 1998.

20 L. J. Farrudia, J. Appl. Crystallogr., 1999, 32, 873.

21 (a) S. Striegler and M. Dittel, Inorg. Chem., 2005, 44, 2728; (b) J. M. Botha, K. Umakoshi, Y. Sasaki and G. J. Lamprecht, Inorg. Chem., 1998, 37, 1609; (c) J. Chen, X. Wang, Y. Zhu, J. Lin, X. Yang, Y. Li, Y. Lu and Z. Guo, Inorg. Chem., 2005, 37,3422 .

22 V. B. Birman, E. W. Uffman, H. Jiang, X. Li and C. J. Kilbane, J. Am. Chem. Soc., 2004, 126, 12226.

23 R. Uson, L. A. Oro and J. A. Cabeza, Inorg. Synth., 1985, 23, 126.

24 T. Irrgang, D. Friedrich and R. Kempe, Angew. Chem., Int. Ed., 2011, 50, 2183.

25 (a) A. Berkessel, T. J. S. Schubert and T. N. Muller, J. Am. Chem. Soc., 2002, 124, 8693; (b) C. Walling and L. Bollyky, J. Am. Chem. Soc., 1964, 86, 3750; (c) C. Walling and L. Bollyky, J. Am. Chem. Soc., 1961, 83, 2968.

26 R. Hartmann and P. Chen, Angew. Chem., 2001, 113, 3693; Angew. Chem., Int. Ed., 2001, 40, 3581. 